\title{
Mex3B: a coreceptor to present dsRNA to TLR3
}

\author{
Cell Research (2016) 26:391-392. doi:10.1038/cr.2016.29; published online 4 March 2016
}

Toll-Like Receptors (TLRs) play critical roles in the early innate immune response to invading pathogens by sensing microorganisms; a number of accessory molecules have been shown to assist microbial recognition by TLRs. In a recent paper in Cell Research, Yang et al. demonstrate that Mex3B is associated with TLR3 in the endosomes and promotes dsRNA binding and proteolytic processing of TLR3, suggesting that Mex3B acts as a coreceptor of TLR3 in response to dsRNA.

Toll-Like Receptors (TLRs), homologues of Drosophila Toll protein, are evolutionarily conserved receptors discovered to be important for host defense against microbial infection [1]. TLRs recognize highly conserved structural motifs known as pathogen-associated molecular patterns (PAMPs), which are exclusively expressed by microbial pathogens, such as lipopolysaccharide (LPS), peptidoglycan (PGN) and lipopeptides from bacterial cell wall, as well as flagellin, bacterial DNA and doublestranded RNA (dsRNA) from viruses. TLRs also recognize danger-associated molecular patterns (DAMPs) that are endogenous molecules released from necrotic cells [1]. TLR3 is involved in recognizing virus-derived dsRNA [2]. Stimulation of TLRs by the corresponding PAMPs or DAMPs initiates signaling cascades leading to the activation of transcription factors, such as nuclear factor- $\kappa \mathrm{B}(\mathrm{NF}-\kappa \mathrm{B})$ and interferon regulatory factors (IRFs). Signaling of all TLRs except TLR3 consists of two distinct pathways: a MyD88-dependent pathway that leads to the production of inflammatory cytokines, and a MyD88- independent pathway associated with the stimulation of interferons. TLR3 utilizes TRIF to mediate its signaling [3].

A number of accessory molecules have been shown to assist microbial recognition by TLRs. For example, LPS is extracted from the bacterial membrane by the LPS-binding protein (LBP), after which it is transferred to CD14. Subsequent transfer from CD14 to an additional accessory molecule MD-2 then allows TLR4-mediated LPS recognition [4, 5]. HMGB1 can also mediate LPS transfer to CD14 to initiate a TLR4-mediated pro-inflammatory response [6]. For TLR3, the structure of the extracellular domain of TLR3 was revealed by crystallography studies as a large horseshoe-shape [7]. It has been shown that CD14, scavenger receptor class-A and clathrin-mediated endocytic pathways participate in cellular uptake of extracellular dsRNA [8-10]. However, how endocytosed dsRNA is presented to TLR3 in intracellular compartments is still poorly understood.

In a recent paper in Cell Research [11], the authors found that an RNAbinding protein Mex3B is involved in this process. Mex3B is associated with TLR3 in the endosomes and promotes the dsRNA binding and proteolytic processing of TLR3 through its own RNAbinding ability, and consequently helps TLR3 activate downstream signals.

Initially, from a cDNA array containing 352 expression clones of ubiquitinrelated proteins, the authors identified an RNA-binding protein Mex3B as a positive regulator of TLR3-mediated signaling using IFN- $\beta$ promoter reporter assay in 293-TLR3 stable cell line. By in vitro overexpression and knockdown experiments in 293-TLR3 stable cells, they showed that Mex3B was involved in poly (I:C)-induced, TLR3-mediated signaling, but not $\mathrm{SeV}$-induced signaling. To further confirm their observation, they generated Mex3B-deficient mice. Consistently, poly(I:C)-induced IRF3 and I $\kappa \mathrm{B} \alpha$ phosphorylation and IFN $-\beta$ production were impaired in $\mathrm{Mex}^{3 \mathrm{~B}^{-/-}}$MEFs, BMDMs or BMDCs, while $\mathrm{SeV}$-, VSV- and EMCV-induced signalings were not affected. In vivo, they also observed less IFN- $\beta$ and $\mathrm{TNF} \alpha$ production in the serum and less mortality in $\mathrm{Mex}_{3} \mathrm{~B}^{-/}$mice challenged with poly(I:C). These data suggest that Mex3B is specifically required and important for TLR3-mediated but not RLR-mediated signaling.

The authors then investigated how Mex3B facilitates TLR3 signaling. They constructed a series of truncation and point mutants of Mex3B and TLR3 to determine the binding and functional domains. Through co-IP experiments and IFN- $\beta$ promoter reporter assay with these truncations, the authors found that two RNA-binding $\mathrm{KH}$ domains in Mex3B and proteolytic cleavage of TLR3 are indispensable for Mex3B to potentiate TLR3-mediated signaling. The authors also demonstrated that Mex3B and TLR3-mediated poly (I:C)induced signaling requires endosomal acidification. By immunofluorescence confocal microscopy, they found that Mex3B and TLR3 colocalized with the endosome marker EEA1, though overexpressing both Mex3B and TLR3 in $293 \mathrm{~T}$ cells may not be a perfect reflection of an endogenous situation with physiologic protein levels. Proteinase $\mathrm{K}$ protection assay also suggests that 
Mex3B and TLR3 are in the lumen of the endosomes.

The author further analyzed whether Mex3B physically interacts with TLR3 and the mechanism whereby Mex3B participates in TLR3 signaling. By endogenous co-IP experiments in cells treated with poly (I:C), the authors found that Mex3B is associated with TLR3, and bound to TRIF in a poly (I:C) signaling-dependent manner. Further, to test whether Mex3B affects the cleavage of TLR 3 , the authors overexpressed or knocked down Mex3B in 293 cells transfected with TLR3, and found that Mex3B promotes TLR3 processing. Lastly, the authors tested whether the RNA-binding protein Mex3B modulated the dsRNA-binding ability of TLR3. By performing biotin-labeled poly (I:C) pull-down experiments, they found that full-length Mex3B bound to poly (I:C) directly and facilitated the binding of TLR3 to poly (I:C), while dominantnegative form of Mex3B, which lacks RNA-binding activity, could not bind to poly (I:C) and inhibited the binding of TLR3 to poly (I:C). These results suggest that the RNA-binding ability of Mex3B is required for the facilitation of ligand binding of TLR 3 and initiation of downstream signals.

Taken together, in this interesting study, the authors identified Mex3B as a coreceptor of TLR3 in mediating virus dsRNA-induced innate immune response. The proposed model is: by sensing viral infection, TLR3 is transported from ER to endosomes where it is cleaved and the stable form of cleaved TLR3 represents the sensor to the dsRNA from virus. The RNA-binding protein Mex3B promotes ligand binding and proteolytic cleavage of TLR3 in endosomes, resulting in full activation of TLR3 and subsequently TLR3-mediated interferon and cytokine induction. This study, together with other reports that described the coreceptors of TLRs, highlighted the requirement of a number of distinct co-receptors and accessory molecules for individual ligands.

Shu Zhu' ${ }^{1}$, Geng Wang ${ }^{1}$,
Xuqiu Lei ${ }^{1}$, Richard A Flavell ${ }^{1,2}$

${ }^{I}$ Department of Immunobiology, Yale University School of Medicine, New Haven, CT 06520, USA; ${ }^{2}$ Howard Hughes Medical Institute, Yale University, New Haven, CT 06520, USA

Correspondence: Richard A Flavell

E-mail: richard.flavell@yale.edu

\section{References}

1 Medzhitov R. Nat Rev Immunol 2001; 1:135-145.

2 Alexopoulou L, Holt AC, Medzhitov R, et al. Nature 2001; 413:732-738.

3 Akira S, Takeda K. Nat Rev Immunol 2004; 4:499-511.

4 Jiang Z, Georgel P, Du X, et al. Nat Immunol 2005; 6:565-570.

5 Shimazu R, Akashi S, Ogata H, et al. J Exp Med 1999; 189:1777-1782.

6 Youn JH, Oh YJ, Kim ES, et al. J Immunol 2008; 180:5067-5074.

7 Choe J, Kelker MS, Wilson IA. Science 2005; 309:581-585.

8 Lee HK, Dunzendorfer S, Soldau K, et al. Immunity 2006; 24:153-163.

9 Itoh K, Watanabe A, Funami K, et al. J Immunol 2008; 181:5522-5529.

10 Limmon GV, Arredouani M, McCann KL, et al. FASEB J 2008; 22:159-167.

11 Yang Y, Wang SY, Huang ZF, et al. Cell Res 2016; 26:288-303. 